\title{
A NOTE ON THE TRACE FORMULAS FOR ALMOST COMMUTING OPERATORS
}

\author{
DAOXING XIA
}

(Communicated by Palle E. T. Jorgensen)

\begin{abstract}
A formula related to the cyclic cohomology for the trace of the product of commutators is established.
\end{abstract}

\section{INTRODUCTION}

Let $X$ and $Y$ be two subalgebras (or groups) in an algebra $\mathbf{A}$ satisfying the condition that

$$
\left[x^{1}, y^{1}\right] \cdots\left[x^{k}, y^{k}\right] \in J \text { for } x^{j} \in X, y^{j} \in Y,
$$

where $[\cdot, \cdot]$ is the commutator, $J$ is the trace ideal of $\mathbf{A}$, and $k$ is a fixed natural number. Let $\tau$ be the trace on $J$. Define functions

$$
\phi_{n}\left(x^{0}, \ldots, x^{n} ; y^{0}, \ldots, y^{n}\right)=\tau\left(\left[x^{0}, y^{0}\right] \cdots\left[x^{n}, y^{n}\right]\right)
$$

for $n \geq k-1$ and $\psi_{n}\left(x^{0}, \ldots, x^{n} ; y^{0}, \ldots, y^{n}\right)=\tau\left(x^{0} y^{0}\left[x^{1}, y^{1}\right] \cdots\left[x^{n}, y^{n}\right]\right)$ for $n \geq k$.

The following are some notations in cycle cohomology [1]. Let $C^{n}$ be the space of functions $f_{n}\left(x^{0}, \ldots, x^{n}\right), t$ be the operation $t: C^{n} \rightarrow C^{n}$ for every $n$

$$
\left(t f_{n}\right)\left(x^{0}, \ldots, x^{n}\right)=(-1)^{n} f_{n}\left(x^{n}, x^{0}, \ldots, x^{n-1}\right),
$$

and $C_{\lambda}^{n}=\left\{f \in C^{n} ; t f=f\right\}$. Let

$$
A f_{n}=\left(1+t+\cdots+t^{n}\right) f_{n} \text { for } f_{n} \in C^{n} ;
$$

then $A: C^{n} \rightarrow C_{\lambda}^{n}$. Let $b$ be the Hochschild boundary operation

$\left(b f_{n}\right)\left(x^{0}, \ldots, x^{n+1}\right)=\left(b^{\prime} f_{n}\right)\left(x^{0}, \ldots, x^{n+1}\right)+(-1)^{n+1} f\left(x^{n+1} x^{0}, x^{1}, \ldots, x^{n}\right)$, where

$$
\left(b^{\prime} f_{n}\right)\left(x^{0}, \ldots, x^{n+1}\right)=\sum_{j=0}^{n}(-1)^{j} f_{n}\left(x^{0}, \ldots, x^{j} x^{j+1}, \ldots, x^{n}\right)
$$

Received by the editors February 18, 1991; presented to the Annual Meeting of the American Mathematical Society in January 1991 and GPOTS, May 1991.

1980 Mathematics Subject Classification (1985 Revision). Primary 47A55; Secondary 47G05.

Key words and phrases. Almost commuting operators, commutators, trace formulas, cyclic cohomology.

This work is supported in part by an NSF grant. 
Let $p$ be the operation

$$
p f=\sum_{j=0}^{n}(n-j) t^{j} f \text { for } f \in C^{n},
$$

where $t^{0}=1$. Define $Z_{\lambda}^{n}=\left\{f \in C_{\lambda}^{n}: b f=0\right\}$ and $B_{\lambda}^{n}=b C_{\lambda}^{n-1}$. Define $S=b p b^{\prime}$. The restriction of $2 \pi i S$ at $Z_{\lambda}^{n}$ coincides with Connes's $S$ operator (cf. [1]), and this operator $S$ also coincides with the constant multiple of some operator in [2].

In this paper the tensor product spaces (for example, $C^{n} \otimes C^{n}$ or $C_{\lambda}^{n} \otimes C_{\lambda}^{n}$ ) of functions of several variables $x^{j} \in X$ and $y^{j} \in Y$ are considered. The operations $t_{x}, A_{x}, b_{x}, \ldots$ or $t_{y}, A_{y}, b_{y}, \ldots$ are the corresponding operations $t, A, b, \ldots$ with respect to $x$ or $y$ respectively. It is easy to see that $t_{x} \phi_{n}=$ $t_{y}^{-1} \phi_{n} ;$ therefore, $A_{x} \phi_{n}=A_{y} \phi_{n}$, and it is denoted by $A \phi_{n}$.

We prove the following.

Theorem. For $n \geq k$, there is a $\Theta_{n} \in C_{\lambda}^{n} \otimes C_{\lambda}^{n}$ such that

$$
A \phi_{n+1}=b_{x} b_{y} \Theta_{n}+\hat{\phi}_{n+1} \text {, }
$$

where

$$
\hat{\phi}_{2 m+p}=(-1)^{m} \prod_{j=1}^{2 m-1}(k+j)^{-1} S_{x}^{m} S_{y}^{m} \phi_{p}
$$

or

$$
\hat{\phi}_{p+2 m}=(-1)^{m} \prod_{j=1}^{2 m}(k+j)^{-1} S_{x}^{m} S_{y}^{m} A \phi_{p}
$$

for $p=k-1, k$. Besides, the function $\Theta_{n}$ is expressed by $\psi_{n}, \ldots, \psi_{k}$, $\phi_{n-1}, \ldots$, and $\phi_{k-1}$.

The explicit form of $\Theta_{n}$ is given in (4), (5), and (38). This theorem is a generalization and refinement of the previous work [3].

The method of the proof of the theorem in $\S 2$ is the same as in [3], but the part of the proof in $\S 3$ is completely new. In order to make this note readable, we will rewrite some formulas in [3].

\section{BASIC LEMMA}

Define

$$
\xi_{n}\left(x^{0}, x^{1}, \ldots, x^{n} ; y^{1}, \ldots, y^{n}\right)=\tau\left(x^{0}\left[x^{1}, y^{1}\right] \cdots\left[x^{n}, y^{n}\right]\right)
$$

and

$$
\eta_{n}\left(x^{1}, \ldots, x^{n} ; y^{0}, y^{1}, \ldots, y^{n}\right)=\tau\left(y^{0}\left[x^{1}, y^{1}\right] \cdots\left[x^{n}, y^{n}\right]\right) .
$$

Let $q$ be the operation

$$
q f=n A f / 2-p t f, \quad f \in C^{n},
$$

for $n>0$ and $q f=0$ for $f \in C^{0}$. Let $r$ be the operation

$$
r f=r_{n}(t) f, \quad f \in C^{n},
$$


for $n>0$, where $r_{n}(\cdot)$ is a polynomial of degree $\leq n-1$ satisfying

$$
q f=(1-t) r f \text { for } f \in C^{n} \text {. }
$$

Besides, $r f=0$ for $f \in C^{0}$. It is easy to see that $r_{x} \phi_{n}=r_{y} \phi_{n}$, and it is denoted by $r \phi_{n}$.

Define $\Theta_{k-2}=\Theta_{k-1}=0$. Define

$$
\zeta_{n}=\psi_{n}-\frac{1}{n} b_{x} b_{y} r \phi_{n-1}-\frac{1}{n(n-1)^{2}} S_{x} S_{y} \Theta_{n-2}
$$

and

$$
\Theta_{n}=\frac{1}{n+1} A_{x} A_{y} \zeta_{n}
$$

for $n \geq k$. By the way, here the function $\Theta_{n}$ is the corresponding $\Theta_{n}$ in [3] multiplied by $n+1$. Define

$$
\hat{\xi}_{k}=\xi_{k}-\frac{1}{k} b_{x} q_{y} \phi_{k-1}, \quad \hat{\eta}_{k}=\eta_{k}-\frac{1}{k} q_{x} b_{y} \phi_{k-1}
$$

and

$$
\hat{\xi}_{n+1}=\frac{1}{(n+1)^{2}} S_{x} A_{y} \hat{\eta}_{n}, \quad \hat{\eta}_{n+1}=-\frac{1}{(n+1)^{2}} A_{x} S_{y} \hat{\xi}_{n}
$$

for $n \geq k$ by mathematical induction.

\section{Define}

$$
\hat{\zeta}_{n}=\frac{1}{(n+1)^{2}} A_{x} p_{y} b_{y}^{\prime} \hat{\xi}_{n}+\frac{1}{n+1} p_{x} b_{x}^{\prime} \hat{\eta}_{n}, \quad n=k, k+1, \ldots
$$

Later we will prove that

$$
\hat{\zeta}_{n}=\frac{1}{(n+1)^{2}} p_{x} b_{x}^{\prime} A_{y} \hat{\eta}_{n}+\frac{1}{n+1} p_{y} b_{y}^{\prime} \hat{\xi}_{n} .
$$

Define

$$
\hat{\phi}_{n+1}=\frac{1}{n+1} S_{x} b_{y} A_{y} \hat{\eta}_{n}, \quad n=k, k+1, \ldots
$$

From (8), (9), $b_{x} \hat{\xi}_{n}=0$, and $b_{y} \hat{\eta}_{n}=0$, it is easy to see that

$$
\hat{\phi}_{n+1}=\frac{1}{n+1} S_{y} b_{x} A_{x} \hat{\xi}_{n} .
$$

By the way, the formulas for $\hat{\xi}_{n}, \hat{\eta}_{n}$, and $\hat{\phi}_{n}$ in [3] are incorrect for $n \geq 5$.

Lemma 1. For $n \geq k$,

$$
\begin{gathered}
A \phi_{n+1}=b_{x} b_{y} \Theta_{n}+\hat{\phi}_{n+1} \\
\zeta_{n}=\frac{1}{n+1}\left\{\Theta_{n}+\frac{1}{n}\left(b_{x} p_{y} b_{y}^{\prime}-p_{x} b_{x}^{\prime} b_{y}\right) \Theta_{n-1}+q_{y} \phi_{n}\right\}+\hat{\zeta}_{n} \\
\xi_{n}=\frac{1}{n}\left(b_{x} \Theta_{n-1}-\frac{1}{n-1} S_{x} b_{y} \Theta_{n-2}+b_{x} q_{y} \phi_{n-1}\right)+\hat{\xi}_{n} \\
\eta_{n}=\frac{1}{n}\left(-b_{y} \Theta_{n-1}-\frac{1}{n-1} b_{x} S_{y} \Theta_{n-2}+q_{x} b_{y} \phi_{n-1}\right)+\hat{\eta}_{n} .
\end{gathered}
$$


Proof. Similar to Lemma 2 of [3], we have

$$
\left(1-t_{x}\right) \psi_{n}=b_{x}^{\prime} \eta_{n}+\phi_{n}
$$

and

$$
\left(1-t_{y}\right) \psi_{n}=b_{y}^{\prime} \xi_{n}-t_{y} \phi_{n}
$$

for $n \geq k$. From (16) and (17), we have

$$
A_{x} \psi_{n}=(n+1) \psi_{n}-p_{x}\left(b_{x}^{\prime} \eta_{n}+\phi_{n}\right)
$$

and

$$
A_{y} \psi_{n}=(n+1) \psi_{n}-p_{y}\left(b_{y}^{\prime} \xi_{n}-t_{y} \phi_{n}\right)
$$

for $n \geq k$. By (18) and (19), we get

(20) $A_{x} A_{y} \psi_{n}=(n+1)^{2} \psi_{n}-(n+1) p_{y} b_{y}^{\prime} \xi_{n}-p_{x} b_{x}^{\prime} A_{y} \eta_{n}-(n+1) q_{y} \phi_{n}$

$$
=(n+1)^{2} \psi_{n}-(n+1) p_{x} b_{x}^{\prime} \eta_{n}-A_{x} p_{y} b_{y}^{\prime} \xi_{n}-(n+1) q_{y} \phi_{n},
$$

since $t_{y} q_{x} \phi_{n}=-q_{y} \phi_{n}$.

On the other hand, from Lemma 1 of [3], we have

$$
\xi_{n+1}=b_{x} \psi_{n} \text { and } \eta_{n+1}=-t_{x} b_{y} \psi_{n}
$$

for $n \geq k$. From (22), we have

$$
b_{x} A_{x} A_{y} \psi_{n}=(n+1)^{2} \xi_{n+1}-S_{x} A_{y} \eta_{n}-(n+1) b_{x} q_{y} \phi_{n}
$$

and

$$
A_{x} b_{y} A_{y} \psi_{n}=-(n+1)^{2} \eta_{n+1}-A_{x} S_{y} \xi_{n}+(n+1) b_{y} q_{x} \phi_{n},
$$

since $b_{x} \xi_{n}=0, b_{y} \eta_{n}=0$, and $t_{x} q_{y} \phi_{n}=-q_{x} \phi_{n}$. From Lemma 3 of [3], we have

$$
A \phi_{n}=b_{y} A_{y} \xi_{n}, \quad A \phi_{n}=-b_{x} A_{x} \eta_{n}
$$

for $n \geq k$. Applying $A_{y}$ to both sides of (23) and $A_{x}$ to both sides of (24), we get

$$
b_{x} A_{x} A_{y} \psi_{n}=(n+1) A_{y} \xi_{n+1}-S_{x} A_{y} \eta_{n}
$$

and

$$
A_{x} b_{y} A_{y} \psi_{n}=-(n+1) A_{x} \eta_{n+1}-A_{x} S_{y} \xi_{n}
$$

since $b_{x} A \phi_{n}=b_{y} A \phi_{n}=0$ (cf. (25)). Therefore,

$$
b_{x} A_{x} b_{y} A_{y} \psi_{n}=(n+1) A \phi_{n+1}-S_{x} b_{y} A_{y} \eta_{n}
$$

and

$$
S_{x} b_{y} A_{y} \eta_{n}=b_{x} A_{x} S_{y} \xi_{n}
$$

From (6), it follows that (14) and (15) hold for $n=k$. Assume that (15) holds for $n \geq k$. Then by the calculation (cf. (26), (27), (28), and (29) in [3]), we have

$$
\begin{aligned}
S_{x} b_{y} A_{y} \eta_{n}= & -\frac{1}{n(n-1)^{2}} b_{x} A_{x} S_{x} b_{y} A_{y} S_{y} \Theta_{n-2} \\
& -\frac{1}{n} b_{x} A_{x} b_{x} b_{y} A_{y} b_{y} r \phi_{n-1}+S_{x} b_{y} A_{y} \hat{\eta}_{n}
\end{aligned}
$$


From (4), (5), (10), (26), and (28), we may prove that (12) holds. By (14), (15), and (27), we may prove (11). From (4), (5), (8), (14), (15), and (21), we have

$$
\begin{aligned}
{\left[(n+1)^{2}-A_{x} A_{y}\right] \psi_{n}-(n+1) q_{y} \phi_{n} } \\
=\frac{(n+1)}{n}\left[b_{x} p_{y} b_{y}^{\prime}-p_{x} b_{x}^{\prime} b_{y}\right] \Theta_{n-1} \\
\quad+\frac{1}{n(n-1)^{2}}\left[(n+1)^{2}-A_{x} A_{y}\right] S_{x} S_{y} \Theta_{n-2} \\
\quad+\frac{1}{n}\left[(n+1)^{2}-A_{x} A_{y}\right] b_{x} b_{y} r \phi_{n-1}+(n+1)^{2} \hat{\zeta}_{n} .
\end{aligned}
$$

This implies (13). Using (20) instead of (21), we may prove (9).

By (4), (13), and (22), we may prove that (7), (14), and (15) hold, where $n$ is replaced by $n+1$, since $b_{x} \hat{\xi}_{n}=0$ and $b_{y} \hat{\eta}_{n}=0$. Therefore, Lemma 1 is proved by mathematical induction.

\section{Calculation of $\hat{\phi}_{n}$}

In this section, we will calculate $\hat{\phi}_{n}$.

Lemma 2. If $f \in C_{\lambda}^{n}$ then

$$
b A S f=(n+1) S b f .
$$

Proof. By $b^{\prime}(1-t)=(1-t) b$, we have

$$
b^{\prime} b f=-\frac{1}{(n+1)}(1-t) S f
$$

(cf. [3, Lemma 4]). Therefore,

$$
\begin{aligned}
S b f=b p b^{\prime} b f & =-\frac{1}{n+1} b p(1-t) S f \\
& =\frac{1}{n+1} b(A-(n+3)) S f=\frac{1}{n+1} b A S f .
\end{aligned}
$$

Lemma 3. $b_{y} A_{y} \eta_{k}=-S_{y} \phi_{k-1}$ and $b_{x} A_{x} \xi_{k}=-S_{x} \phi_{k-1}$.

Proof. By direct calculation, it is easy to see that

$$
\left(1-t_{y}\right) \eta_{k}=b_{y}^{\prime} \phi_{k-1}
$$

therefore,

$$
t_{y}^{m} \eta_{k}=\eta_{k}-\left(1+t_{y}+\cdots+t_{y}^{m-1}\right) b_{y}^{\prime} \phi_{k-1}
$$

for $m=1,2, \ldots, k$. Thus,

$$
A_{y} \eta_{k}=(k+1) \eta_{k}-p_{y} b_{y}^{\prime} \phi_{k-1}
$$

which proves that $b_{y} A_{y} \eta_{k}=-S_{y} \phi_{k-1}$. Similarly, we prove the other equality in Lemma 3.

Corollary 1. If $m \geq 1$, then

(32) $b_{y}\left(A_{y} S_{y}\right)^{m-1} A_{y} \hat{\eta}_{k}=-\prod_{j=1}^{m-1}(k+2 j-1) S_{y}^{m-1}\left[S_{y} \phi_{k-1}+\frac{1}{k} q_{x} b_{y} A_{y} b_{y} \phi_{k-1}\right]$, 
where $\prod_{j=1}^{0}(k+2 j-1)$ is replaced by 1 and

$$
S_{x}^{m} \phi_{k-1} \in C_{\lambda}^{k+2 m-1} \otimes C^{k-1} .
$$

Proof. By Lemma 3 and (6), equality (32) holds for $m=1$. Assume that (32) holds for some $m \geq 1$. Then by Lemma 2 we have

$$
\begin{aligned}
b_{y}\left(A_{y} S_{y}\right)^{m} A_{y} \hat{\eta}_{k} & =(k+2 m-1) S_{y} b_{y}\left(A_{y} S_{y}\right)^{m-1} A_{y} \hat{\eta}_{k} \\
& =-\prod_{j=1}^{m}(k+2 j-1) S_{y}^{m}\left[S_{y} \phi_{k-1}+\frac{1}{k} q_{x} b_{y} A_{y} b_{y} \phi_{k-1}\right] .
\end{aligned}
$$

Thus, (32) holds for every $m \geq 1$ by mathematical induction. From (32) it is easy to see that

$$
S_{y}^{m} \phi_{k-1} \in C^{k-1} \otimes C_{\lambda}^{k+2 m-1} .
$$

Changing $\hat{\eta}_{k}$ to $\hat{\xi}_{k}$, similar to (34), we may prove (33).

Corollary 2. For $m \geq 0$,

$\hat{\phi}_{k+2 m+1}$

$$
=(-1)^{1+m} \prod_{j=1}^{2 m+1}(k+j)^{-1}\left[S_{x}^{m+1} S_{y}^{m+1}-\frac{1}{k} S_{x}^{m} b_{x} A_{x} b_{x} S_{y}^{m} b_{y} A_{y} b_{y} r\right] \phi_{k-1} .
$$

Proof. From (7), it is easy to see that

$$
\hat{\eta}_{n+2}=-\frac{1}{(n+2)^{2}(n+1)^{2}} A_{x} S_{x} S_{y} A_{y} \hat{\eta}_{n}
$$

for $n \geq k$. By (10), we have

$$
\hat{\phi}_{k+2 m+1}=(-1)^{m}(k+2 m+1)^{-1} \prod_{j=1}^{2 m}(k+j)^{-2}\left(S_{x} A_{x}\right)^{m} S_{x} b_{y}\left(A_{y} S_{y}\right)^{m} A_{y} \hat{\eta}_{k} .
$$

Using (32) and (36), we get

$$
\begin{aligned}
\hat{\phi}_{k+2 m+1}= & (-1)^{m+1} \prod_{j=1}^{2 m+1}(k+j)^{-1} \prod_{j=1}^{m}(k+2 j-1)^{-1}\left(S_{x} A_{x}\right)^{m} S_{x} \\
& \times S_{y}^{m}\left[S_{y} \phi_{k-1}+\frac{1}{k} q_{x} b_{y} A_{y} b_{y} \phi_{k-1}\right] .
\end{aligned}
$$

By (33), we have $\prod_{j=1}^{m}(k+2 j)^{-1}\left(S_{x} A_{x}\right)^{m} S_{x} \phi_{k-1}=S_{x}^{m+1} \phi_{k-1}$. From $b^{\prime}(1-t)=$ $(1-t) b$, we get

$$
S_{x} q_{x} \phi_{k-1}=b_{x} p_{x}\left(1-t_{x}\right) b_{x} r \phi_{k-1}=-b_{x} A_{x} b_{x} r \phi_{k-1}
$$

therefore, (37) implies (35). From (31), we have

$$
\begin{aligned}
S b A b f & =b p b^{\prime} b A b f=-(n+2)^{-1} b p(1-t) S A b f \\
& =-(n+2)^{-1} b(n+4-A) S A b f=(n+2)^{-1} b A b p b^{\prime} A b f
\end{aligned}
$$

for $f \in C^{n}$. Thus,

$$
S^{m} b A b f=\prod_{j=1}^{m}(n+2 j)^{-1} b A b\left(p b^{\prime} A b\right)^{m} f
$$


for $f \in C^{n}$. Hence,

$$
\begin{aligned}
& S_{x}^{m} b_{x} A_{x} b_{x} S_{y}^{m} b_{y} A_{y} b_{y} r \phi_{k-1} \\
& \quad=\prod_{j=1}^{m}(k+2 j)^{-2} b_{x} A_{x} b_{x}\left(p_{x} b_{x}^{\prime} A_{x} b_{x}\right)^{m} b_{y} A_{y} b_{y}\left(p_{y} b_{y}^{\prime} A_{y} b_{y}\right)^{m} r \phi_{k-1} .
\end{aligned}
$$

From (39), we only have to change $\Theta_{k+2 m}$ in (1) to get

$$
\begin{aligned}
\Theta_{k+2 m}= & \frac{1}{k+2 m} A_{x} A_{y} \zeta_{k+2 m}+(-1) \prod_{j=0}^{m+1}(k+2 j)^{-2} \prod_{n=1}^{m}(k+2 n-1)^{-2} \\
& \times A_{x} b_{x}\left(p_{x} b_{x}^{\prime} A_{x} b_{x}\right)^{m} A_{y} b_{y}\left(p_{y} b_{y}^{\prime} A_{y} b_{y}\right)^{m} r \phi_{k-1} .
\end{aligned}
$$

Then (34) reduces to (2) for $p=k-1$. Since $(k-A) \phi_{k-1}=\left(1-t_{x}\right)\left(1-t_{y}\right)$ $\times u\left(t_{x}\right) \phi_{k-1}$ for some $u$, we may prove (3) for $p=k-1$.

To prove (2) for $p=k$, instead of Lemma 3, we have to establish the following.

Lemma 4. $b_{y} A_{y} \hat{\eta}_{k+1}=-\frac{1}{k+1} S_{y} A \phi_{k}$ and $b_{x} A_{x} \hat{\xi}_{k+1}=-\frac{1}{k+1} S_{x} A \phi_{k}$.

Proof. By $b^{\prime}(1-t)=(1-t) b,(7)$, and (25), we have

$$
\begin{aligned}
\left(1-t_{y}\right) \hat{\eta}_{k+1} & =-\frac{1}{(k+1)^{2}} A_{x}\left(1-t_{y}\right) b_{y} p_{y} b_{y}^{\prime} \hat{\xi}_{k} \\
& =-\frac{1}{(k+1)^{2}} A_{x} b_{y}^{\prime}\left(k+1-A_{y}\right) b_{y}^{\prime} \hat{\xi}_{k}=\frac{1}{(k+1)^{2}} A_{x} b_{y}^{\prime} b_{y} A_{y} \hat{\xi}_{k} \\
& =\frac{1}{(k+1)^{2}} A_{x} b_{y}^{\prime} A \phi_{k}-\frac{1}{k(k+1)^{2}} A_{x} b_{x} b_{y}^{\prime} b_{y} A_{y} q_{y} \phi_{k-1} \\
& =\frac{1}{k+1} b_{y}^{\prime} A \phi_{k},
\end{aligned}
$$

since $A_{y} q_{y} \phi_{k-1}=0$. Using the method in the proof of Lemma 3, we may prove Lemma 4.

By the same method in the proof of (2) and (3) for $p=k-1$, we may prove (2) and (3) for $p=k$.

\section{REFERENCES}

1. A. Connes, Non-commutative differential geometry, Inst. Hautes Études Sci. Publ. Math. 62 (1985), 257-360.

2. D. Xia, Cyclic cohomology associated with differences operators and anticommutators, Integral Equations Operator Theory 10 (1987), 739-750.

3. __ Trace formulas for almost commuting operators, cyclic cohomology and subnormal operators, Integral Equations Operator Theory 14 (1991), 276-298.

Department of Mathematics, Vanderbilt University, Nashville, Tennessee 37235 